\title{
Energy aware routing and traffic management for software defined networks
}

\author{
Berna Özbek, Yiğitcan Aydoğmuş \\ Electrical and Electronics Engineering \\ Izmir Institute of Technology \\ Izmir, Turkey \\ \{bernaozbek,yigitcanaydogmus\}@iyte.edu.tr
}

\author{
Aydın Ulaş, Burak Görkemli,Kazım Ulusoy \\ ARGELA A.Ş. \\ Istanbul, Turkey \\ \{aydin.ulas,burak.gorkemli,kazim.ulusoy\}@argela.com.tr
}

\begin{abstract}
Since traffic diversity and volume increase with growing popularity of mobile applications, there is the strong need to manage the traffic carried by networks. Software defined networks can simplify network management while enabling new services by employing traffic management including routing whose goal is to maximize the given utility while satisfying capacity requirements. In this paper, we propose an efficient routing algorithm to minimize the cost based on power consumption determined by the number of active OpenFlow switches in a software defined network while satisfying throughput requirements of all flows according to constraints on link capacities in the network. We evaluate the performance of the proposed algorithm based on the number of active switches for different network topologies with various scenarios.
\end{abstract}

\section{INTRODUCTION}

Software defined networking (SDN) makes the network control plane an independent software platform and it is suitable for sophisticated environments [1][2]. The control plane of SDN is centralized and sends the control information to the data plane. This architecture provides a view of the entire network enabling the controller to optimize the whole network. One of the potential main components of the SDN is OpenFlow switch which is the first standard transmission interface defined between the control and forwarding layers of an SDN architecture [3].

Traffic management is an important mechanism to optimize the performance of the network by dynamically analyzing, predicting and regulating the behavior of the data to achieve routing and power saving [4]. SDN provides great incentive for novel traffic management techniques that exploit the global network view, status, and flow patterns/characteristics. One of the goal of traffic management is to decide how to route traffic in a network in order to balance several objectives such as maximizing throughput, balancing the link utilization, minimizing the power consumption, controlling the bandwidth allocated to competing flows and minimizing latency.

There are several traffic management strategies examined for SDNs. In [5], a weighted multipath algorithm has been presented in which each router splits the traffic regarding to delay-sense weighted Equal-Cost Multipath split rule, so that overall network end-to-end delay is optimized. In [6], a fair model has been designed by considering the network resources

978-1-4673-9486-4/16/\$31.00 (c) 2016 IEEE based on bandwidth and flow table while minimizing the delay for multiple control applications in SDN. The distributed solution associated optimization of flow-based end-to-end Qualityof-Services (QoS) has been presented in [7]. A low-complexity hybrid routing scheme to achieve near-optimal load balancing for multiple traffic matrices has been given in [8]. In [9], the flows are scheduled separately in time domain by using exclusive routing (EXR) which guarantees that one link can occupied at most one flow at a time. In addition to that, EXR provides flexibility to define priorities to flows. The three-tier virtual machine placement algorithm which combines energy efficiency and QoS awareness has been suggested in [10].

In addition to them, the power saving strategies have been considered to perform energy efficient traffic management for SDN. In [11], an integrated control scheme which combines smart sleeping and power scaling algorithms has been given by testing in data center with Fat-Tree topology under low and high traffic cases. In [12], an optimization method for rule placement considering link capacities and space constraints on routers has been suggested to reduce energy consumption for a backbone network. In [13], an energy efficient routing scheme with traffic aggregation for each flow to decrease the number of active network devices while maintaining acceptable performance has been presented. In [14], how to optimize power management globally at network level has been considered by re-routing traffic through different paths to adjust the load of links when the network is relatively idle. In [15], the energy saving mechanism has been presented by turning on a minimal amount of network devices to carry the traffic, instead of powering on all switches at all time by formulating as an MILP problem and presenting an heuristic algorithm with four different strategies. In [16], a pre-established multi-path model has been described for energy-aware routing. In [17], a energy efficient controller association algorithm has been given by switching off the maximum number of links by taking into account delay, link and controller load constraints.

In this paper, we propose an energy efficient routing and traffic management algorithm to minimize the cost based on energy consumption determined as the number of active OpenFlow switches in the network. This goal is not always achieved by just minimizing the number of hops, which sometimes forces to power on new switches. Therefore, we propose a 
low complexity algorithm that achieves an efficient routing in terms of energy saving while satisfying the throughput requirement of all flows.

The contribution beyond state of the arts is to design an energy efficient routing algorithm which has almost the same complexity as shortest path algorithm to become suitable for practical applications and to achieve a better energy saving performance. We evaluate the performance of proposed algorithm on energy consumption by considering different throughput requirements of flows in various network topologies with given link capacities.

In Section II, we give the network model, formulate the optimization problem and introduce GA. In Section III, we present the architecture which includes the proposed low complexity routing solution. The performance evaluations and the conclusion are given in Section IV and Section V, respectively.

\section{NETWORK MODEL}

The SDN can be modeled as a graph $G<\mathbb{V}, \mathbb{Z}>$ where $\mathbb{V}$ is the set of switches and $\mathbb{Z}$ is the set of possible links between the switches through available ports. $N_{u, t}$ links can be established between node $u$ and node $t$ proportional to the number of ports in the nodes. Each link $\left(u_{y}, t_{y}\right) \in \mathbb{Z}$ has a capacity $C_{\left(u_{y}, t_{y}\right)}$ where $y=1, \ldots, N_{u, t}$. The capacity of each link is adjusted according to the number of available ports in the switches and the maximum available capacity, $C_{(u, t)}$. $\mathbb{F}$ is the set of $K$ flows and each flow $f_{k} \in \mathbb{F}$ has several parameters, source, $\mathrm{s}_{f_{k}}$, destination, $\mathrm{d}_{f_{k}}$ and throughput requirement, $R_{f_{k}}$.

The aim of our solution is to find the set of routes $\mathbf{p}=$ $\left\{\mathrm{p}_{f_{1}}, \mathrm{p}_{f_{2}}, \ldots, \mathrm{p}_{f_{K}}\right\}$ where $\mathrm{p}_{f_{k}}$ is the set of routes composed of the active links through source to destination belonging to the flow $f_{k}$. While finding the routes $\mathbf{p}$ that satisfies throughput requirements of all flows, we reduce the power consumption based on the number of active switches in the network. Let $x_{v} \in\{0,1\} ; \forall v$ indicate whether the switch $v$ is active or not. If the switch $v$ is used through the links selected for the route $\mathbf{p}$, then its indicator, $x_{v}$, becomes 1 .

The total number of active OpenFlow switches in the network is determined by

$$
T(\mathbf{p})=\sum_{v \in \mathbb{V}} x_{v}(\mathbf{p})
$$

In order to achieve energy awareness based on the number of active switches in the network, the optimization problem is defined as,

$$
\min T(\mathbf{p})
$$

subject to

$$
\begin{gathered}
C_{\left(u_{y}, t_{y}\right) \in \mathrm{p}_{f_{k}}} \geq R_{f_{k}} \quad \forall f_{k} \\
\sum_{y=1}^{N_{u, t}} C_{\left(u_{y}, t_{y}\right)} \leq C_{(u, t)} \quad \forall u, t
\end{gathered}
$$

The first constraint states that the link capacity must be equal to or higher than throughput requirement of each assigned flow. The second constraint denotes that the total link capacity, $C_{u, t}$ between switch $u$ and $t$ must be equal to or higher than demanding rate of total established $N_{u, t}$ links.

Since we consider the nighttime traffic case in which the network load is low, the overall network capacity is higher than the rate requirements of all flows. Hence it is possible to shut down a certain number of OpenFlow switches.

\section{PROposed SOLUTION}

We propose an efficient routing algorithm having almost the same complexity as shortest-path algorithm. In order to implement the proposed flow based energy efficient routing in SDN, we present the architecture illustrated in Figure 1.

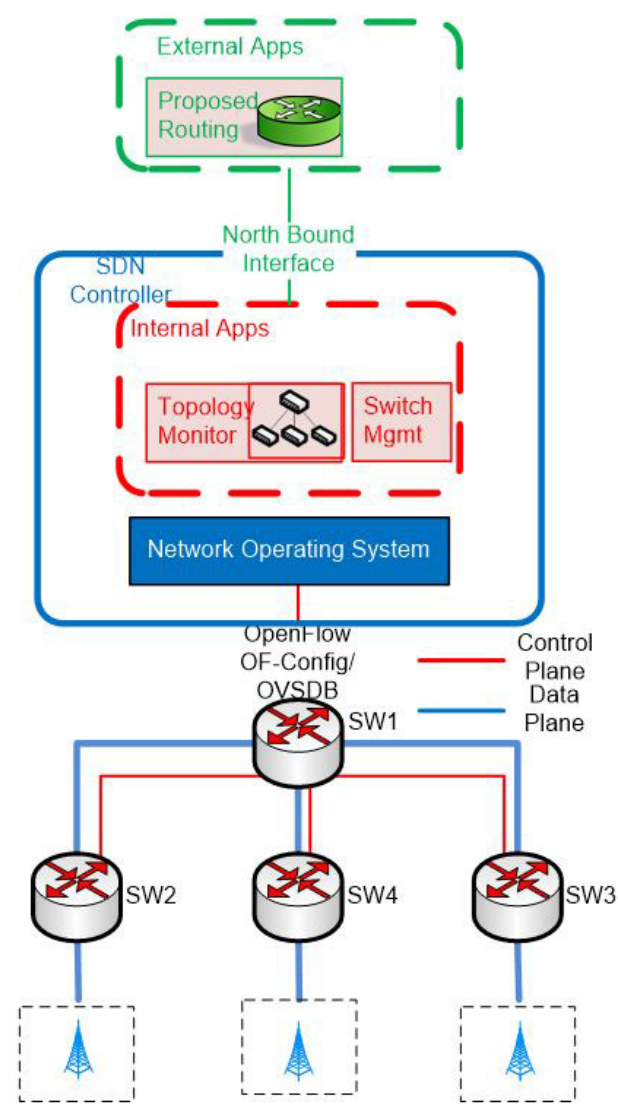

Fig. 1: The proposed architecture

The efficient routing module (referred to as "Proposed Routing" in the architecture) which is connected to a North Bound agent implementing the application programming interface (API), receives the initial topology and the capacity of the links from the SDN Controller. When a request for a path between two nodes is received, the controller relays this request to the application and the proposed algorithm that will be explained below finds the route and returns back to the controller to set up the route (via OpenFlow or any suitable south bound protocol). The proposed algorithm also communicates with the switch management module (referred to as Switch Mgmt) to shut down and turn on switches to reduce energy consumption. 
We formulate the problem as an energy routing with baremetal switches and we determine the gain on energy saving based on the number of active switches which is directly corresponding to power consumption. Besides that the problem also has roots on the Network function virtualization (NFV) side where enabling/disabling switches correspond to instantiating (or allocating servers) for Virtual Machines (therefore also leading to power consumption) which will act as virtual Switches.

In the proposed low complexity approach, the flows are routed sequentially in a random manner instead of routing simultaneously. This method also allows for real-time operation where flow requests are arrived and then are released.

In the proposed algorithm, the optimization problem is defined for each flow $f_{k} \in \mathbb{F}$ by,

$$
\min T\left(\mathrm{p}_{f_{k}}\right)
$$

subject to

$$
C_{\left(u_{y}, t_{y}\right) \in \mathrm{p}_{f_{k}}} \geq R_{f_{k}}
$$

where $T\left(\mathrm{p}_{f_{k}}\right)=\sum_{v \in \mathbb{V}} x_{v}\left(\mathrm{p}_{f_{k}}\right)$.

After routing the flow $f_{y}$, the total available link capacity belonging to all links is updated by,

$$
C_{(u, t)}=C_{(u, t) \in \mathrm{p}_{f_{y}}}-R_{f_{y}}
$$

In order to solve this problem given in Eq. (5), we propose a low complexity routing algorithm. Figure 2 depicts the flow chart of the proposed approach.

The proposed low complexity routing algorithm is described in detail as follows:

- Initialization: The sets of input OpenFlow switches, II, which are connected to SDN controller and the output OpenFlow switches, $\mathbb{O}$, which are connected to such as base stations for the wireless networks are determined in a given network topology. Then, $F$-shortest paths [18] for each input and output pair $(i, o)$ are computed and stored in the set $W_{(i, o)}$ for $\forall i \in \mathbb{I}$ and $\forall o \in \mathbb{O}$. The set of all routes is initialized by $S=\emptyset$.

- For the first flow, $f_{1}$ :

- For the flow $f_{1}$ with source $\mathrm{s}_{f_{1}}$ and destination $\mathrm{d}_{f_{1}}$, chose the optimum route $\mathrm{p}_{f_{1}}$ from the set $W_{\left(\mathrm{s}_{f_{1}}, \mathrm{~d}_{f_{1}}\right)}$ according to Eq.(5) while satisfying Eq.(6). This route corresponds to shortest path solution for flow $f_{1}$ among all possible routes in the set $W_{\left(\mathrm{s}_{f_{1}}, \mathrm{~d}_{f_{1}}\right)}$ since it is the first flow to be routed in the network.

- Update $S=S \cup \mathrm{p}_{f_{1}}$.

- Update the available link capacities in the network by using Eq. (7).

- For $k=2, \cdots, K$

- For flow $f_{k}$, find the $B \leq F$ routes having shortest paths as $\mathrm{W}_{\left(\mathrm{s}_{f_{k}}, \mathrm{~d}_{f_{k}}\right)}^{b} ; b=1, \ldots, B$ while satisfying Eq.(6).

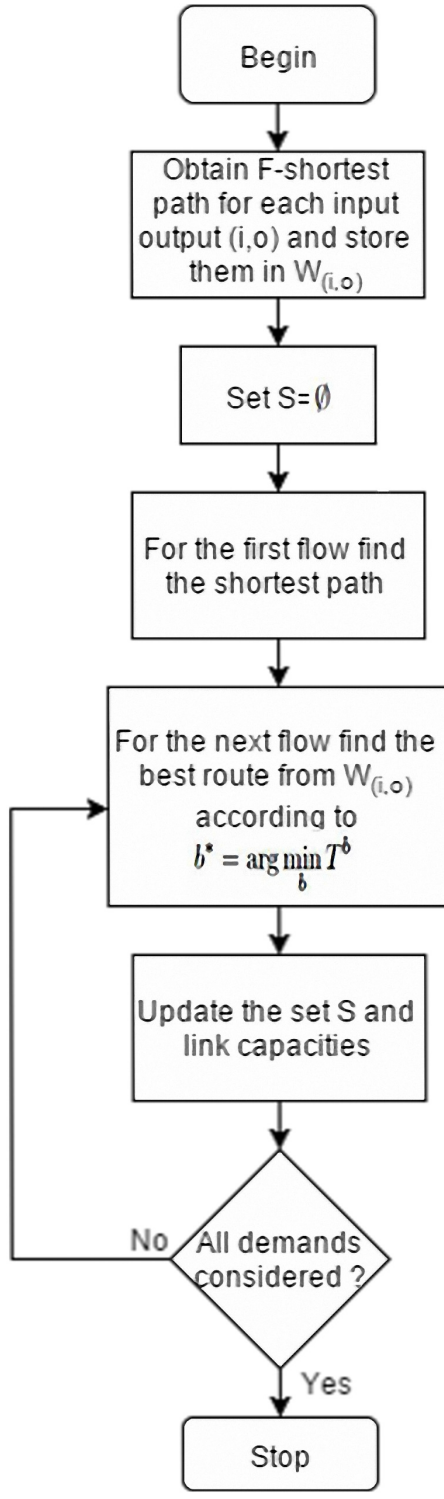

Fig. 2: The flow chart of proposed routing algorithm

- Select the best route for flow $f_{k}$ in terms of total number of active switches while maximizing the switch usage:

$$
b^{*}=\arg \min _{b} T^{b}
$$

where $S^{b}=S \cup \mathrm{W}_{f_{k}}^{b}$ and $T^{b}=\sum_{v \in \mathbb{V}} x_{v}\left(S^{b}\right)$ with $b=1, \ldots, B$.

- Update $S=S \cup \mathrm{p}_{f_{k}}^{b^{*}}$ by adding the new switches that become active for flow $f_{k}$ to the set $S$.

- Update the available link capacities in the network by Eq. (7).

- End.

In any step, if there are more than one route that satisfies the constraints while giving the same number of switches on the cost function, we select the one which has the highest 
remaining available link capacity. The reason is that we will follow this route for the remaining flows in order to avoid to increase the number of active switches.

\section{Performance Evaluations}

In the paper, we consider nighttime traffic in which the network load is low and consequently the number of flows is low. Then, we show that it is possible to shut down a certain number of OpenFlow switches by considering four different network topologies and various scenarios.

The network topology as shown in Figure 3, there are 28 OpenFlow switches with the ports by varying between $2,6,8$ and 14. Then, the total link capacities between two OpenFlow switches are determined depending on the link capacity per port defined in each scenario.

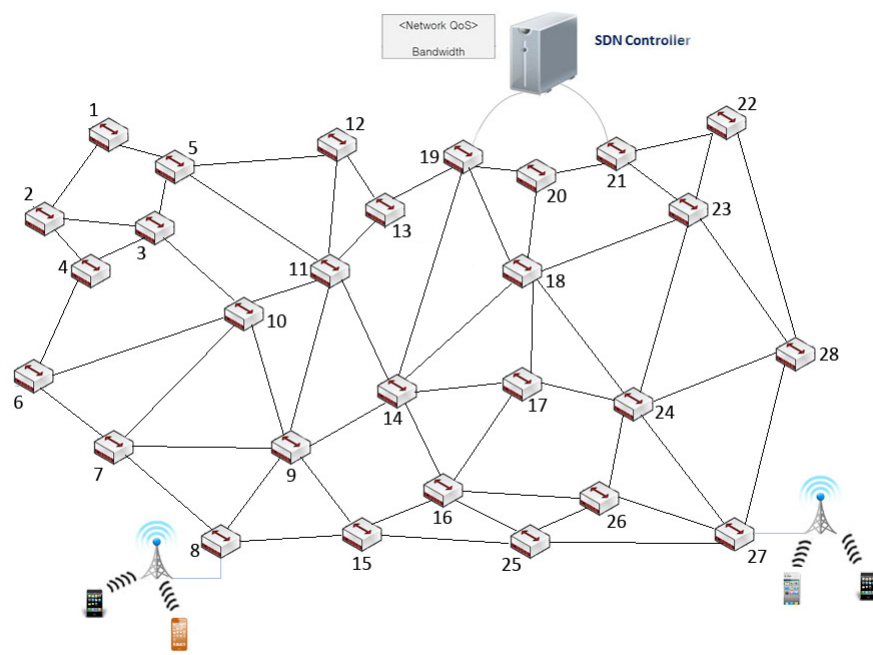

Fig. 3: The network topology

We obtain the performance results according to the following scenarios:

- Scenario 1 (S1): All flows have the same destination and source nodes. The throughput requirements of all flows are the same with $100 \mathrm{Mbps}$. The capacity per port is equal to $100 \mathrm{Mbps}$.

- Scenario 2 (S2): Half of the flows have different source and destination nodes. The throughput requirements of all flows are the same with $100 \mathrm{Mbps}$. The capacity per port is equal to $100 \mathrm{Mbps}$.

As shown in Figure 4, the achievable percentage of power reduction of the proposed routing algorithm is also about $15 \%$ compared to the shortest path algorithm in the second network topology.

We also generate various topologies based on Waxman [19] to illustrate the performance of the proposed algorithm. All links between any two nodes have the capacity of 1 Gbps. In the topology, the network nodes are uniformly placed in the plane and edges defined according to distances between nodes. The probability to have an edge between nodes $u$ and $v$ is given by

$$
P(u, v)=\alpha e^{\frac{d}{\beta L}}
$$

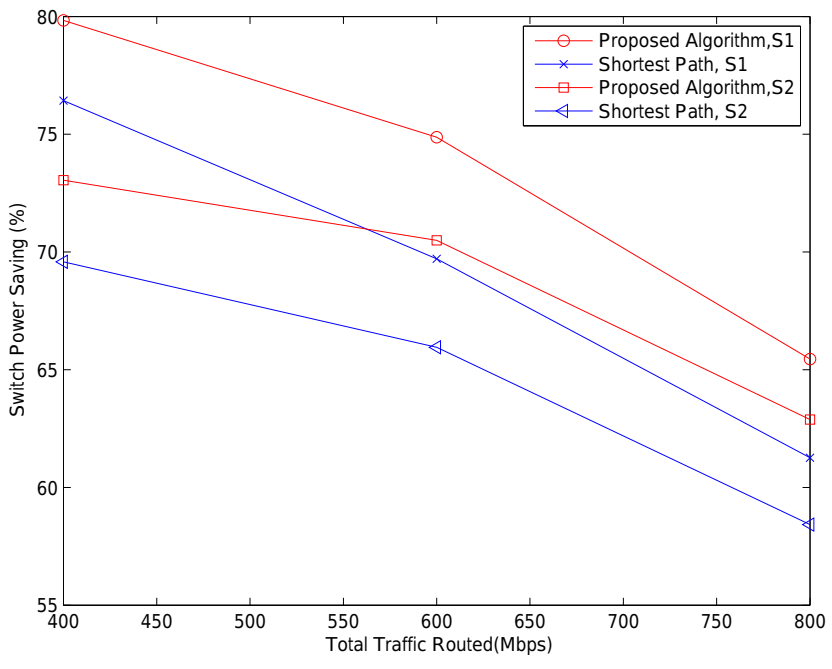

Fig. 4: Performance results for Scenario 1 and Scenario 2.

where $\alpha$ determines the maximal link probability and $\beta$ controls the length of edges, $d$ is the distance between the node $u$ and $v$, and $L$ is the maximum distance between any two nodes. The parameters are given in Table I.

\begin{tabular}{|c|c|c|c|}
\hline \hline Topology & node count & alpha & beta \\
\hline \hline First Waxman Network & 20 & 50 & 0.1 \\
\hline Second Waxman Network & 40 & 50 & 0.1 \\
\hline \hline
\end{tabular}

TABLE I: Parameters for Generation of Waxman Network

In the first Waxman topology, node 12 and node 19 are chosen as the possible sources and node 11 , node 17 and node 18 are selected as the possible destination. The requirements of flows have generated as uniformly distributed demands by 100 Mbps, 200 Mbps and 300 Mbps.

In the second Waxman topology, node 15 and node 36 are chosen as the possible sources and node 6 , node 17 and node 22 are selected as the possible destinations. The requirements of flows have generated as uniformly distributed demands by $100 \mathrm{Mbps}, 300 \mathrm{Mbps}$ and 500 Mbps.

According to the performance results given in Figures 5 and 6, the proposed algorithm gives better performance up to $20 \%$ than the shortest path algorithm.

\section{CONClusion}

We have proposed an architecture including routing algorithm to design energy efficient software defined networks. For the case when the traffic load in the network is low, we have formulated the optimization problem to enable to shut down switches in the network. We have proposed an efficient low complexity approach which routes the flows sequentially while having almost the same complexity as shortest-path algorithm. We have provided the power consumption performances based on the number of active OpenFlow switches 


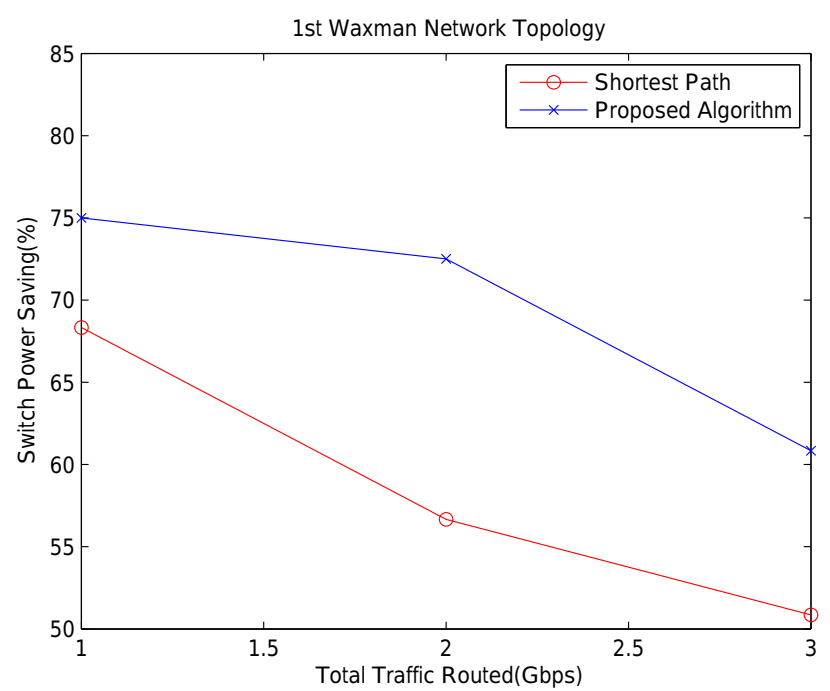

Fig. 5: Performance results for the First Waxman Topology

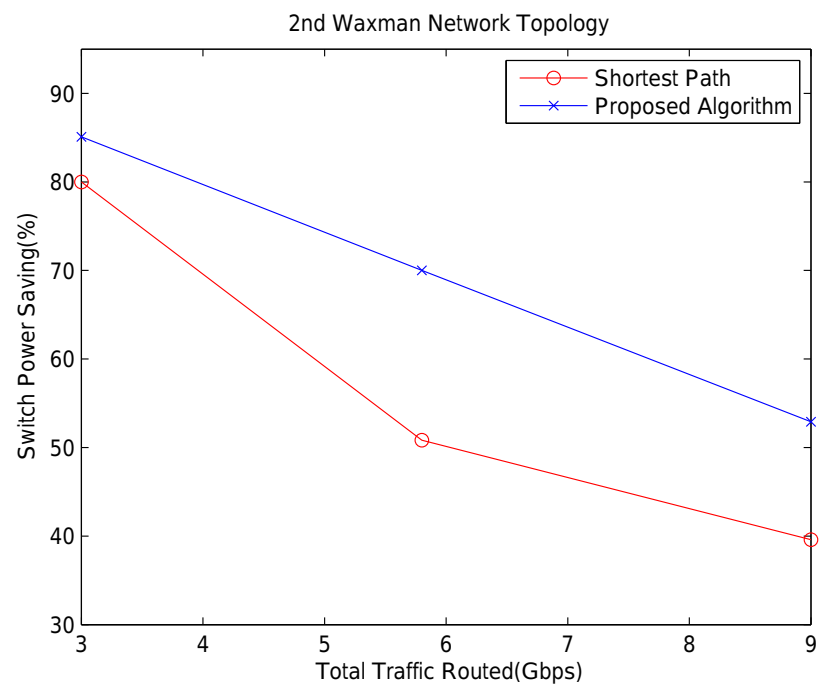

Fig. 6: Performance results for the Second Waxman Topology

for different throughput constraints for considering various network topologies and scenarios. As a future work, we will adapt the proposed architecture to software defined mobile networks by taking into account different parameters such as users' location, users' signal to noise ratio, traffic model and interference level.

\section{ACKNOWLEDGMENT}

This work has been carried out in the framework of CELTIC-Plus project CP2012/2-5 SIGMONA.

\section{REFERENCES}

[1] K.Hyojoon, N. Feamster,Improving network management with software defined networking, IEEE Communication Magazine, vol. 51,pp:114-119, February 2013.
[2] S. Sezer, S. Scott-Hayward, P. Chouhan, B. Fraser, D. Lake, J. Finnegan, N. Viljoen, M. Miller, and N. Rao, Are we Ready for SDN? Implementation Challenges for Software-Defined Networks, IEEE Communications Magazine, vol. 51, no. 7, pp. 36-43, February 2013.

[3] A. Lara, A. Kolasani, and B. Ramamurthy, Network Innovation using OpenFlow: A Survey, IEEE Communications Surveys Tutorials, vol. pp, no. 99, pp. 1-20, 2013.

[4] S. Agarwal, M. Kodialam, and T. Lakshman, Traffic engineering in software defined networks, IEEE 32th Annual Joint Conference of the IEEE Computer and Communications Societies (INFOCOM), pp. 22112219, April 2013.

[5] J. Zhang, K. Xi, L. Zhang, and H. Chao, Optimizing network performance using weighted multipath routing, 21st International Conference on Computer Communications and Networks (ICCCN), pp. 1-7, 2012.

[6] T.Feng, J. Bi, K. Wang, Joint Allocation and Scheduling of Network Resource for Multiple Control Applications in SDN, IEEE Operations and Management Symposium (NOMS), pp.1-7, 2014.

[7] H.E. Egilmez, A.M. Tekalp,Distributed OoS Architectures for Multimedia Streaming Over Software Defined Networks, IEEE Transactions on Multimedia, volume: 16, Issue: 6, pp. 1597 - 1609, 2014.

[8] J. Zhang, K. Xi, M. Luo, H. J. Chao, Load balancing for multiple traffic matrices using SDN hybrid routing, IEEE 15th International Conference on High Performance Switching and Routing, pp. 44-49, 2014.

[9] Dan Li; Yunfei Shang; Congjie Chen, Software defined green data center network with exclusive routing, IEEE INFOCOM, pp.1743,1751, 2014.

[10] S. Wang; Huang, P.P.-W.; Wen, C.H.-P.; Li-Chun Wang, EQVMP: Energy-efficient and QoS-aware virtual machine placement for software defined data center networks, 2014 International Conference on Information Networking (ICOIN), pp.220-225, 10-12 Feb. 2014.

[11] T. Nguyen et al., Modeling and experimenting combined Smart sleep and power scaling algorithms in energy-aware data center networks, Simulation Modeling Practice and Theory, vol. 39, pp. 20-40, 2013.

[12] F.Giroire, J. Moulierac, T. Khoa Phan. Optimizing Rule Placement in SoftwareDefined Networks for Energy-aware Routing. [Research Report] RR-8537, 2014.

[13] S. Oda, D. Nobayashi, Y. Fukuda and T. Ikenaga, Flow-based Routing Schemes for Minimizing Network Energy Consumption using OpenFlow, The Fourth International Conference on Smart Grids, Green Communications and IT Energy-aware Technologies, 2014.

[14] Rui Wang; Zhipeng Jiang; Suixiang Gao; Wenguo Yang; Yinben Xia; Mingming Zhu, Energy-aware routing algorithms in Software-Defined Networks, IEEE 15th International Symposium on World of Wireless, Mobile and Multimedia Networks (WoWMoM), pp.1,6, 19-19 June 2014.

[15] Markiewicz, A, Phuong Nga Tran, Timm-Giel, A., Energy consumption optimization for software defined networks considering dynamic traffic, IEEE 3rd International Conference on Cloud Networking (CloudNet),Luxembourg, October 2014.

[16] Celenlioglu, M.R.; Goger, S.B.; Mantar, H.A., An SDN-based energyaware routing model for intra-domain networks, 22nd International Conference on Software, Telecommunications and Computer Networks (SoftCOM), pp.61-66, 17-19 Sept. 2014.

[17] Ruiz-Rivera, A.; Kwan-Wu Chin; Soh, S., GreCo: An Energy Aware Controller Association Algorithm for Software Defined Networks, IEEE Communications Letters, vol.19, no.4, pp.541,544, April 2015.

[18] J. Yen, Finding the $K$ shortest loopless paths in a network, Theory Series, vol. 17, no. 11 , pp. $712716,1971$.

[19] B. Waxman, Routing of Multipoint Connections, IEEE Journal on Selected Areas in Communications, vol:6, pp.1617 - 1622, 2002. 